\section{A rare presentation of idiopathic right subclavian artery aneurysm successfully tackled by endovascular stent-grafting}

A 32-year-old woman presented with symptoms of dysphagia to solid food for 6 months. She had no significant past medical history or trauma. Examination revealed a subtly palpable pulsatile swelling at the root of right side of the neck. Contrast-enhanced CT showed a complex saccular aneurysm of the proximal right subclavian artery (figure 1A, B). The involved aneurysmal segment contained three separate saccules, the largest of which measured $4.7 \times 2.3 \mathrm{~cm}$. A selective digital subtraction angiography was done contemplating the possibility of endovascular intervention (figure 1C). In a cross-section image, the aneurysm is seen abutting the trachea which in turn compresses the oesophagus (figure 2A). Oesophago-gastroduodenoscopy was normal. Barium swallow showed smooth extrinsic compression of the oesophageal lumen at the level of aneurysm (figure 2B). An extensive aetiological work-up ruled out atherosclerosis, connective tissue disorders, vasculitis, infections, collagen disorders and congenital cardiovascular anomalies. She was taken up for endovascular stent-grafting and the aneurysm was successfully excluded using two stent-grafts (figure $3 \mathrm{~A}, \mathrm{~B}$ ). At follow-up, she has complete resolution of symptoms.

\section{DISCUSSION}

Subclavian artery aneurysm accounts for $<0.2 \%$ of all aneurysms. Atherosclerosis, trauma, infections, vasculitis, connective tissue disorders and collagen vascular disease are causative for more than $95 \%$ of these. ${ }^{1}$ Idiopathic subclavian aneurysms have very rarely been reported and most of them are considered congenital. $^{2}$

The aneurysms are usually detected incidentally, but when symptomatic they commonly present as a pulsatile mass, neck pain, upper-limb ischaemia or mediastinal mass. Rare presentations that have been reported include dyspnoea, dysphagia and
A

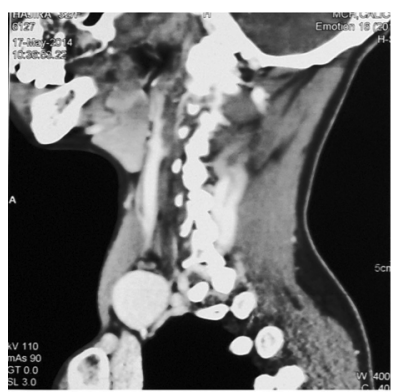

B

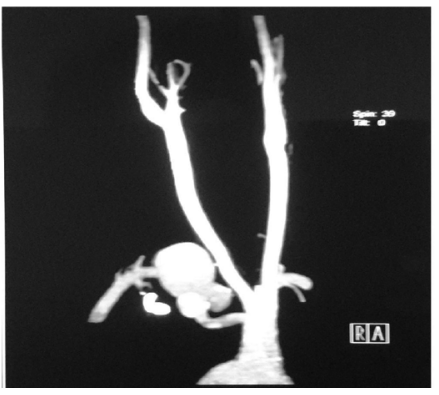

C

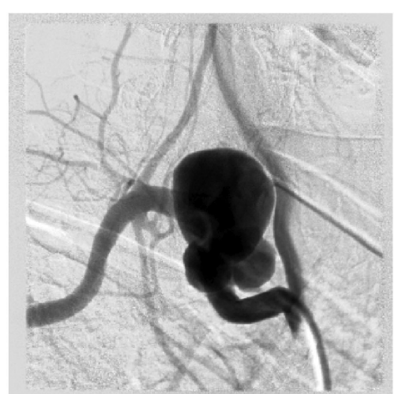

Figure 1 (A) Sagittal section of contrast-enhanced CT of neck showing the complex right subclavian aneurysm. (B) Reconstructed CT angiogram image showing the aortic arch vessels and complex aneurysm at the proximal right subclavian artery. (C) Digital subtraction angiography showing the 3 separate saccules of the complex aneurismal segment.

Figure 2 (A) Horizontal cross-section of contrast-enhanced CT showing the aneurysm pushing the trachea which, in turn, compresses the oesophagus.

(B) Barium swallow image in oblique view showing focal smooth compression of the oesophageal lumen.
A

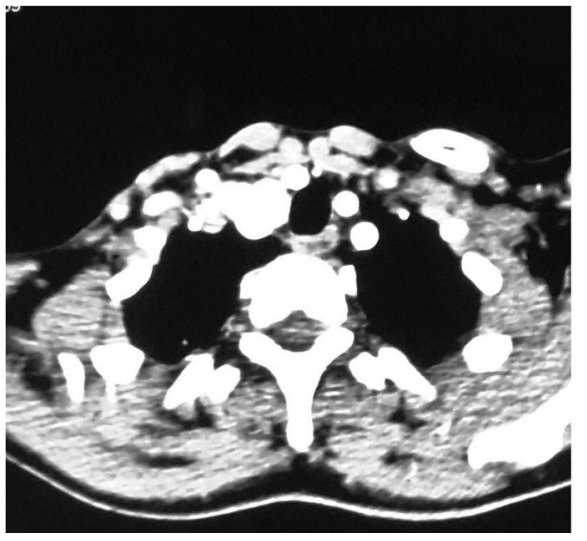

B

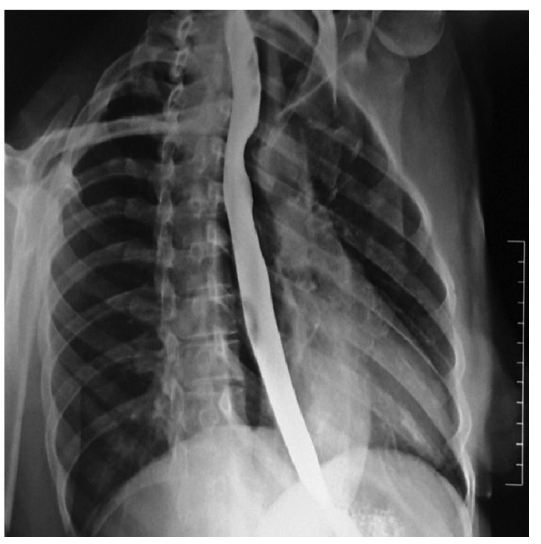


Figure 3 (A) Final angiographic pigtail injection at the ascending aorta after covered stent-graft placement showing complete exclusion of the aneurysm. (B) Multidetector CT reconstructed image poststent graft repair.
A

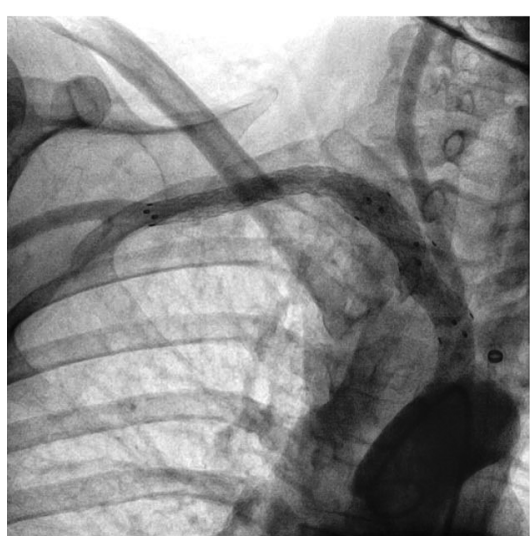

B

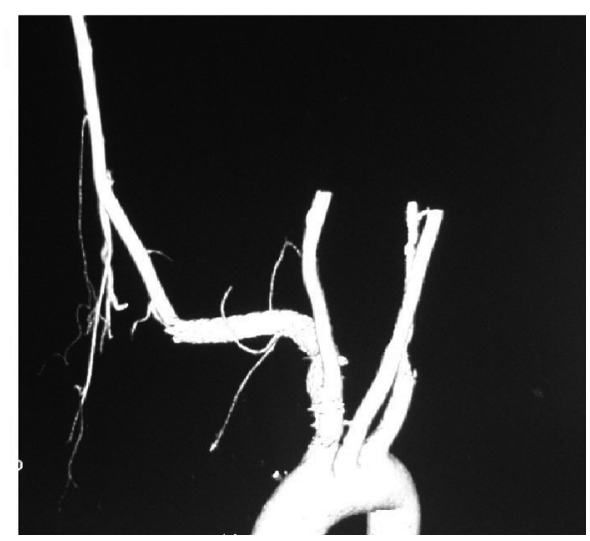

haemoptysis. ${ }^{3}$ Endovascular stent-grafting has been increasingly reported as a management option.

\section{Sandeep Mohanan, Rakesh Jain, M N Krishnan, Vinayakumar Desabandhu} Department of Cardiology, Calicut Government Medical College, Calicut, Kerala, India

Correspondence to Dr Sandeep Mohanan, Department of Cardiology, Calicut Government Medical College, 'Deepthi', Kavu stop, Chevayoor PO, Calicut, Kerala 673017, India; stg222@gmail.com

Acknowledgements The authors would like to acknowledge the effort and support provided by Dr C Bastian and Dr Shajudheen in patient management and care.

Contributors SM has been involved in the evaluation, care and management of the patient. MNK and VD had supervised the evaluation and care of the patient and have significantly contributed in making necessary corrections in the paper. RJ was involved in patient care and manuscript writing.

Competing interests None.
Patient consent Obtained.

Provenance and peer review Not commissioned; internally peer reviewed.

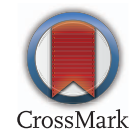

To cite Mohanan S, Jain R, Krishnan MN, et al. Heart Asia 2014;6:150-151. Heart Asia 2014;6:150-151. doi:10.1136/heartasia-2014-010576

\section{REFERENCES}

1 Dougherty MJ, Calligaro KD, Savarese RP, et al. Atherosclerotic aneurysm of the intrathoracic subclavian artery: a case report and review of the literature. J Vasc Surg 1995;21:521-9.

2 Stahl RD, Lawrence PF, Bhirangi K. Left subclavian artery aneurysm: Two cases of rare congenital etiology. J Vasc Surg 1999;29:715-18.

3 Windfuhr JP, Schubert $D$, Remmert $S$. Aneurysm of the subclavian artery. An unusual cause of dysphagia. HNO 2004;52:1097-102. 